\title{
VacSol: a high throughput in silico pipeline to predict potential therapeutic targets in prokaryotic pathogens using subtractive reverse vaccinology
}

Muhammad Rizwan ${ }^{1 \dagger}$, Anam Naz ${ }^{2 \dagger}$, Jamil Ahmad ${ }^{1 *}$, Kanwal Naz $^{2}$, Ayesha Obaid², Tamsila Parveen ${ }^{3}$, Muhammad Ahsan ${ }^{1}$ and Amjad Ali ${ }^{2^{*}}$

\begin{abstract}
Background: With advances in reverse vaccinology approaches, a progressive improvement has been observed in the prediction of putative vaccine candidates. Reverse vaccinology has changed the way of discovery and provides a mean to propose target identification in reduced time and labour. In this regard, high throughput genomic sequencing technologies and supporting bioinformatics tools have greatly facilitated the prompt analysis of pathogens, where various predicted candidates have been found effective against certain infections and diseases. A pipeline, VacSol, is designed here based on a similar approach to predict putative vaccine candidates both rapidly and efficiently.

Results: VacSol, a new pipeline introduced here, is a highly scalable, multi-mode, and configurable software designed to automate the high throughput in silico vaccine candidate prediction process for the identification of putative vaccine candidates against the proteome of bacterial pathogens. Vaccine candidates are screened using integrated, well-known and robust algorithms/tools for proteome analysis, and the results from the VacSol software are presented in five different formats by taking proteome sequence as input in FASTA file format. The utility of VacSol is tested and compared with published data and using the Helicobacter pylori 26695 reference strain as a benchmark.

Conclusion: VacSol rapidly and efficiently screens the whole bacterial pathogen proteome to identify a few predicted putative vaccine candidate proteins. This pipeline has the potential to save computational costs and time by efficiently reducing false positive candidate hits. VacSol results do not depend on any universal set of rules and may vary based on the provided input. It is freely available to download from: https://sourceforge.net/projects/vacsol/.
\end{abstract}

Keywords: Reverse vaccinology, Computational pipeline, Vaccine candidates, Subtractive proteomics, PVCs, VacSol

\footnotetext{
* Correspondence: dr.ahmad.jamil@gmail.com; amjaduni@gmail.com

${ }^{\dagger}$ Equal contributors

${ }^{1}$ Research Center for Modelling and Simulation (RCMS), National University of Sciences and Technology (NUST), H-12, Islamabad, Pakistan

${ }^{2}$ Atta-ur-Rahman School of Applied Biosciences (ASAB), National University of

Sciences and Technology (NUST), H-12, Islamabad, Pakistan

Full list of author information is available at the end of the article
} 


\section{Background}

In silico prediction of vaccine candidates has great significance in various life science disciplines, including biomedical research [1]. The conventional approach of vaccine development requires pathogenic cultivation in vitro that is not always possible. Although this methodology has the potential to produce successful vaccines and has long been in practice, but now considered timeconsuming and inadequate for most pathogens. This caveat is particularly evident when microbes are inactive, protective, or even in the case where antigen expression is decreased; rendering the conventional approach a significant challenge for putative vaccine candidate discovery $[2,3]$. These basic problems have led scientists to develop new vaccinology approaches based on advanced computational tools. In particular, with the introduction of high-throughput sequencing techniques over the last decade and the advent of bioinformatics approaches, Rino Rappouli revolutionized Pasteur's vaccinology procedure by introducing a novel "reverse vaccinology" method [4-6]. This advanced in-silico technique for vaccine prediction couples genomic information and analysis with bioinformatics tools. Using this approach, several vaccines have been successfully developed against microbial pathogens [7-9]. Reverse vaccinology is now recognized as safer and more reliable as compared to conventional vaccinology methods $[10,11]$.

Using the reverse vaccinology approach, various predictive and analytical tools (Vaxign, VaxiJen, JennerPredict) have been designed for the identification of putative vaccine candidates. These tools are widely available online [12-14], but only a handful of softwares and pipelines, like NERVE and Vacceed $[15,16]$, are accessible as full packages. Although web-based pipelines are efficient, their drawbacks include time delays and constraints for input file size.

NERVE (New Enhanced Reverse Vaccinology Environment), a Perl based modular pipeline for in-silico identification of potential vaccine candidates, generates results through text interface configuration and is an efficient, modular-based standalone software for vaccine candidate identification [15]. But it only focuses on adhesion proteins whereas several non-adhesion proteins can also participate in host-pathogen interactions (including porin, flagellin, invasin, etc.), and most of them are pathogenic as well as antigenic. Therefore, there exists a perilous need for an updated and advanced analysis tool that inclusively provides every putative candidate in its output.

Vacceed is another highly configurable architecture designed to perform high throughput in silico identification of eukaryotic vaccine candidates. Vacceed is, in fact, able to reduce false vaccine candidates that are selected for laboratory validation to save time and money [16], but this highly efficient, scalable, and configurable program provides limited information on pathogenicity and putative functional genes. These main parameters prove instrumental in the determination of potential vaccine candidates. Thus, given the current software limitations, we sought to utilize the reverse vaccinology approach to overcome limitations of currently available pipelines.

We therefore focused on in silico reverse vaccinology approach to address the issues that were present in previous pipelines, and to precisely screen out the putative vaccine candidates from whole bacterial genome in silico. We designed a new automated pipeline, termed VacSol, to efficiently screen for the therapeutic vaccine agents from the bacterial pathogen proteome to save both time and resources.

\section{Implementation}

VacSol was designed to screen and detect prioritized proteins as vaccine candidates, and its functionality is presented in Fig. 1. Notably, this software was developed on platform independent Java language, is highly flexible through one executable .jar file, and does not require any software installation. The VacSol functionality does depend on the installation of various tools that are used as pre-requisites for the pipeline execution (such as PSORTb for localization prediction), and we have integrated various freely available, well-performing and updated tools in the VacSol pipeline to achieve optimal performance. VacSol has been tested and analyzed to be fully functional on Ubuntu 12.04 .5 (64 bit) version. It can also work on any operating system with already installed and functional prerequisite tools, given minor modifications. PSORTb [17] and OSDDlinux (http:// osddlinux.osdd.net/) have also been pre-packaged for robust and user-friendly installation (See installation guide).

VacSol also offers the user to select either a single tool (selective) or complete pipeline to predict potential vaccine candidates (PVCs). Protein sequences are subjected to the main analytical process where the input format is validated through the FASTA Validator for vaccine target prediction. This main process is multi-threaded, as one can run as many threads as there are cores available in their system. Further, the pipeline is capable of processing multiple sequences in parallel. The process of sequence prioritization is performed in a number of steps to prioritize the input sequences, and is elaborated in Fig. 2. Each step is forwarded by a special script and protein sequences are screened at every step indistinctly with generated results displayed in various formats. After processing all the sequences of an input file, the 


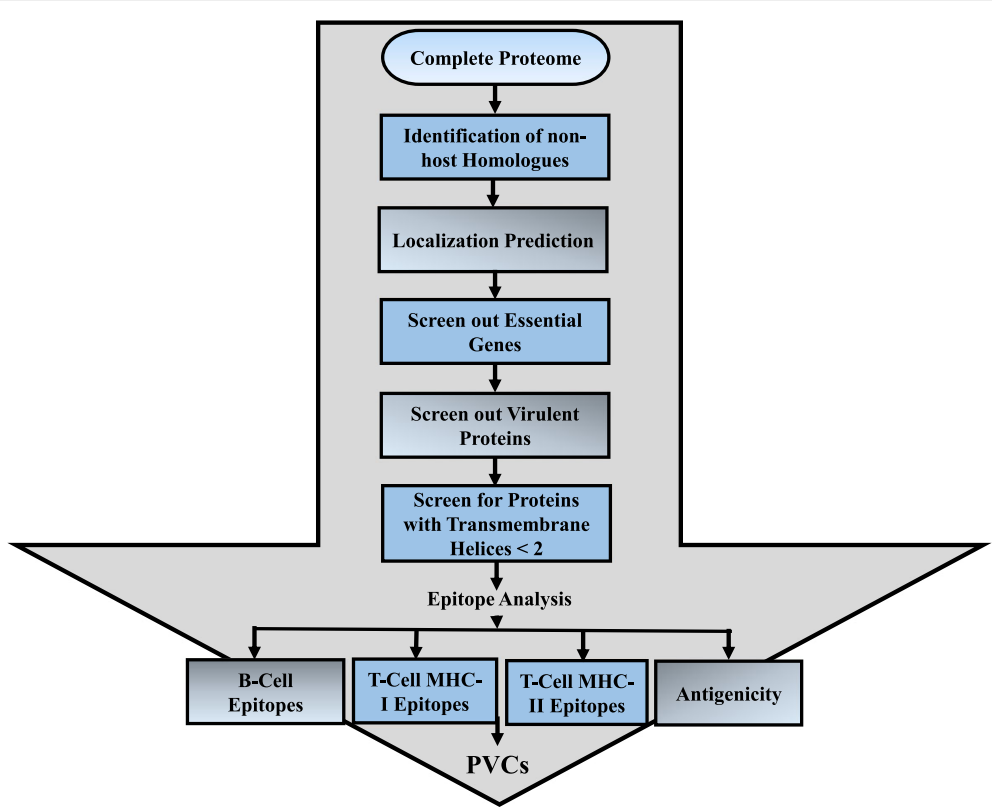

Fig. 1 Schematic diagram of the protein prioritization process. Steps to prioritize proteins to identify PVCs include: (1) the complete bacterial proteome (sequences) subjected to the VacSol pipeline for identifying PVCs; (2) the complete proteome is searched for non-host homologous, essential, virulent proteins residing in the extracellular membrane with less than two transmembrane helices; (3) proteins that meet the selection criteria are considered to be PVC proteins; (4) prioritized proteins are further analyzed for antigenic B- and T-cell epitopes

prioritized sequences are then subjected for epitope mapping. Thereafter, all prioritized sequences are again directed to thread pool processing to generate final results. Final results are engendered in five different formats (FASTA, XML, JSon, HTML, and PDF format), ensuring the expandability and scalability of the designed pipeline for users. Step-wise information of VacSol is provided in a comprehensive user guide (Additional file 1).

\section{Distinct features}

The VacSol interface is designed on four different modules: (i) Blaster, a module for predicting homology using BLASTp; (ii) Localization Predictor, predicting subcellular location; (iii) Helicer, predicting transmembrane helices; and, (iv) Epitoper, a module designed to predict B-cell and T-cell epitopes. These modules function on the basis of implemented tools (Table 1) required to screen prioritized proteins

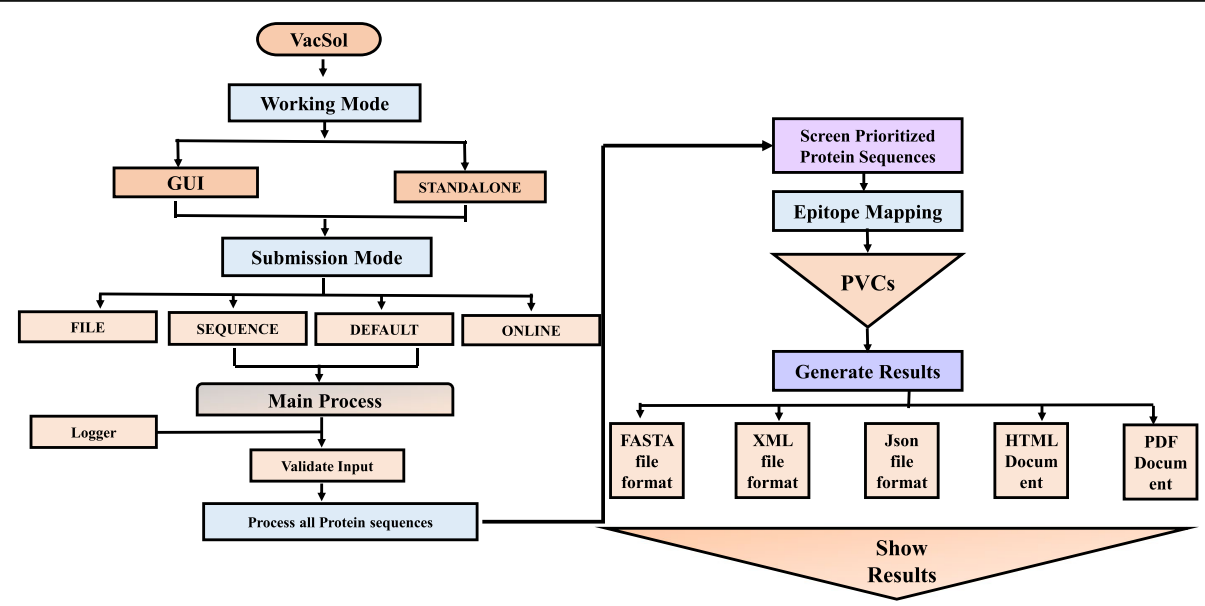

Fig. 2 Schematic diagram of VacSol processing. VacSol is comprised of two working modes: (i) GUI, and (ii) Standalone. The software is highly flexible as it permits users to submit a FASTA proteome sequence in four different ways. Submitted input is validated through a FASTA validator, and then VacSol screens the whole proteome to prioritize proteins that have antigenic B- and T-cell epitopes. Individual tool results (Localizer, Blaster, Helicer, and Epitoper) and complete results are generated for prioritized proteins in five different formats 
Table 1 Tools and databases integrated and implemented in VacSol

\begin{tabular}{lll}
\hline Name & Function & Source(S) \\
\hline BLAST+2.2.25-7 & New command line sequence alignment application developed using the NCBI C++ toolkit. & {$[38]$} \\
Pftools2.3 & Package of programs that support the search method of generalized profile formatting. & {$[39]$} \\
PSORTb3.0 & Protein subcellular localization prediction tool. & {$[17]$} \\
HMMTOP 2.0 & Transmembrane topology prediction tool. & {$[32]$} \\
DEG 10.0 & Database of essential genes. & {$[28]$} \\
VFDB & Virulence factors database. & {$[31]$} \\
ABCPred & B-Cell epitope prediction tool. & {$[40]$} \\
Propred-I & Prediction of promiscuous major histocompatibility complex (MHC) Class-l binding sites. \\
Propred & Prediction of MHC Class-II binding regions in an antigen sequence. & {$[41]$} \\
UniProt-SwissProt & Manually annotated protein sequences database with information extracted from literature.
\end{tabular}

(targets). The VacSol pipeline is developed in Java, a platform independent language [18].

\section{Results}

Test data

VacSol performs various proteome-wide analyses and generates results in five different formats. This pipeline was validated using a sample data set of the Helicobacter pylori proteome. The selected strain of H. pylori 26695 (RefSeq NC_000915.1) is comprised of 1576 proteins or coding regions [19], and the whole proteome was scanned in each protein prioritizing step.

\section{Implementation of VacSol for test data}

The first working step was performed by identifying the non-host homologs, required to elute host homologous proteins to restrict the chance of autoimmunity [20,21]. Out of 1576 possible proteins, 1452 were screened as non-human homologous proteins by using BLASTp against RefSeq [22] and SwissProt [23] databases. For BLAST non-human homologs, criteria included a Bit Score $>100$, E-Value $<1.0 \mathrm{e}^{(-5)}$, and percentage identity $>35 \%$ [24]. Next, these 1452 proteins were subjected for further protein prioritization processing by VacSol to predict subcellular localization. 65 proteins were found to be in the secretome and exoproteome, of which 23 proteins lie in the extracellular region, and 42 were screened as outer-membrane proteins. Prioritization of proteins according to localization substantially contributed to enhance the PVCs identification process [25]. Surface exposed proteins tend to be involved in pathogenesis, making them prime targets as vaccine candidates [26]. Similarly, both extracellular and secreted proteins are readily accessible to antibodies as compared to intracellular proteins, and therefore represent ideal vaccine candidates. Results obtained through PSORTb, and integrated in VacSol, were then cross-checked with CELLO2GO [27] to confirm the localization of putative candidate proteins. After localization validation, screened proteins were checked for their essentiality. 667 proteins were sorted as essential genes required for the survival of gastric pathogen $H$. pylori. Finally, 10 proteins have been prioritized following all the criteria. This analysis reduced the cost and time of PVCs identification by excluding proteins with no suitable features for further processing.

The Database of Essential Genes (DEG) [28] was then used to predict essential genes. Results demonstrated that all 10 of the prioritized proteins were essential proteins, thus making them putative vaccine candidates. In the next step, the proteome was screened for virulent proteins, as identification of virulent factors in essential proteins is a key step in the vaccine development process [29]. Essential genes of a pathogen tend to be virulent, substantiating these checks as key factors in the prediction of target proteins to prioritize vaccine candidates $[21,30]$. In our case, 267 proteins were found to be virulent proteins among whole proteome of the pathogen.

VFDB [31] results, coinciding with our pipelinegenerated results, demonstrated that all prioritized proteins contained virulence factors, concluding that these 10 proteins are potential vaccine targets. Next, proteins were checked for their transmembrane topology. VacSol explored 1254 proteins with less than 2 transmembrane helices, as these proteins are often deemed the best candidates. Having more than one transmembrane helix in a protein makes expression and colonization difficult, and multiple transmembrane helices fail to purify recombinant proteins for vaccine development [21]. HMMTOP version 2.0 [32] was applied to enumerate transmembrane helices with default parameter values. Subsequently, proteins were checked for their functional annotation from UniProt (Table 2) [33]. UniProt characterizes functionality of proteins based on sequence and/ or similarity with functionally annotated proteins [23]. Insight into the role of targeted proteins in a system provides a detailed understanding as to how putative targets 
Table 2 Functional annotation of prioritized proteins

\begin{tabular}{|c|c|c|c|c|c|c|}
\hline $\begin{array}{l}\text { Protein ID } \\
\text { (VacSol) }\end{array}$ & Bacterial protein & $\begin{array}{l}\text { Gene symbol } \\
\text { (NCBI) }\end{array}$ & $\begin{array}{l}\text { Molecular weight } \\
\mathrm{kDa} \text { (ExPASy) }\end{array}$ & $\begin{array}{l}\text { Molecular function } \\
\text { (UNIPROT) }\end{array}$ & Domains (Interpro Scan) & $\begin{array}{l}\text { Trans-membrane } \\
\text { Helices }\end{array}$ \\
\hline 3 & $\begin{array}{l}\text { Iron(III) dicitrate transport } \\
\text { protein (FecA) }\end{array}$ & HP1400 & 94.827 & Receptor activity & $\begin{array}{l}\text { TonB-dependent receptor } \\
\& \text { plug domain }\end{array}$ & 0 \\
\hline 285 & Flagellin A (FlaA) & HP0601 & 53.287 & $\begin{array}{l}\text { Cell motility, Signal } \\
\text { transduction and } \\
\text { structural molecule } \\
\text { activity }\end{array}$ & $\begin{array}{l}\text { Flagellin, Flagellin_D0/D1, } \\
\text { Flagellin_hook_IN_motif }\end{array}$ & 0 \\
\hline 534 & Putative beta-lactamase & HP1098 & 31.594 & Beta-lactamase activity & $\begin{array}{l}\text { Sel1-like, TPRlike__ } \\
\text { helical_dom, TPR_2 }\end{array}$ & 0 \\
\hline 825 & $\begin{array}{l}\text { Iron(III) dicitrate transport } \\
\text { protein (FecA) }\end{array}$ & HP0807 & 88.946 & Receptor activity & $\begin{array}{l}\text { TonB-dependent receptor } \\
\& \text { plug domain }\end{array}$ & 0 \\
\hline 837 & Flagellin B (FlaB) & HP0115 & 53.882 & $\begin{array}{l}\text { Structural molecule } \\
\text { activity }\end{array}$ & Flagellin, Flagellin_D0/D1 & 0 \\
\hline 907 & $\begin{array}{l}\text { Toxin-like outer } \\
\text { membrane protein }\end{array}$ & HP0289 & 311.288 & Not defined & $\begin{array}{l}\text { Autotransport_beta\& } \\
\text { Vacuolating_cytot oxin_put }\end{array}$ & 1 \\
\hline 995 & $\begin{array}{l}\text { Toxin-like outer } \\
\text { membrane protein }\end{array}$ & HP0922 & 274.563 & Not defined & $\begin{array}{l}\text { VacA2 (motif), } \\
\text { Autotransporte_beta, } \mathrm{PbH} 1\end{array}$ & 0 \\
\hline 982 & Beta-lactamase HcpA & HP0211 & 27.366 & $\begin{array}{l}\text { Peptidoglycan, cell } \\
\text { wall synthesis }\end{array}$ & Sel1-like, TPRlike_helical_dom & 0 \\
\hline 1184 & $\begin{array}{l}\text { Toxin-like outer } \\
\text { membrane protein }\end{array}$ & HP0610 & 212.964 & Not defined & $\begin{array}{l}\text { Vacuolating cytotoxin putative \& } \\
\text { Autotransporter beta domain }\end{array}$ & 0 \\
\hline 1359 & $\begin{array}{l}\text { Iron(III) dicitrate transport } \\
\text { protein (FecA) }\end{array}$ & HP0686 & 87.698 & Receptor activity & $\begin{array}{l}\text { TonB-dependent receptor, } \\
\text { betabarrel, plug domain }\end{array}$ & 0 \\
\hline
\end{tabular}

can be used to reduce pathogen burden and virulence. Prioritized proteins included 3 homologs of FecA (HP1400, HP0807, HP0686), FlaA (HP0601), FlaB (HP0115), HcpA (HP0211), HcpC (HP1098), and toxinlike outer membrane proteins (HP0289, HP0610, and HP0922). B-cell and T-cell epitopes screened for prioritized candidates along with their features (location, score, no. of MHC I \& II binding alleles) have been shown in results file (Additional file 2).

An overview of the results displayed by VacSol are shown in Fig. 3. Each protein sequence was assigned a unique VacSol ID for retrieval, and the overall results for $H$. pylori are provided as Additional file 2. The total duration of these analyses was $90 \mathrm{~min}$, on a machine with 2GB RAM.

\section{Discussion}

The prioritized putative vaccine targets against $H$. pylori 26695 included FecA (HP1400), FecA (HP0807), FecA (HP0686), FlaA (HP0601), FlaB (HP0115), HcpA (HP0211), HcpC (HP1098), and toxin-like outer membrane proteins (HP0289, HP0610, and HP0922). Among these target candidates, Iron (III) dicitrate transport protein, FecA (HP1400, HP0807, and HP0686), interacts with $\operatorname{Ton} B$, a protein involved in the virulence process. Previous studies have shown that controlled and mutated TonB leads to increased immunization [34]. Indeed, by targeting HP1400, HP0807, and HP0686, TonB can be controlled, making these three promising putative vaccine candidates.
Flagelline proteins (flaA and flaB) are responsible for the pro-inflammation of gastric mucosa that leads to the development of gastric/peptic ulcers, making flaA and flaB considerable candidates for novel vaccine development [35]. Likewise, Betalactamase $H c p A$ and $H c p C$ are highly pathogenic proteins that are directly involved in different infections caused by $H$. pylori [36]. The HcpA protein is also involved in bacterial and eukaryotic host interaction [37]. These protein annotations verify that VacSol limited its screening to the proteins that are biologically relevant putative and therapeutic vaccine candidates.

Previous studies have linked three toxin-like proteins with virulent proteins and vaccine candidates $B a b A$, CagS, Cag6, HpaA, and VacA [21]. Indeed, Cag proteins are also well-known pathogenic proteins, involved in pathogenic pathways, while the $H c p A$ protein has been shown to be involved in bacterial and eukaryotic host interactions [37]. Using our computational approach, we have designed the VacSol pipeline to further the field of vaccinology by reducing time, cost and trial burdens in novel putative vaccine candidate protein identification. Proteins predicted using this pipeline against $H$. pylori strain may serve as promising PVCs against gastric pathogens, as substantiated by previous findings in the literature. Further evaluation of these PVCs can lead to the development of novel and effective vaccines against $H$. pylori. 


\begin{tabular}{|c|c|c|c|c|c|c|}
\hline \multicolumn{7}{|c|}{ Summary Report } \\
\hline Seq. Id & Non-Homologous & Localization & Essential & Virulent & Helices $<2$ & Annotated \\
\hline \multirow[t]{2}{*}{1} & $\odot$ & 8 & $\theta$ & 8 & $\theta$ & $\theta$ \\
\hline & 0 & Cytoplasmic & 16 & 0 & OUT: 0 & \\
\hline \multirow[t]{2}{*}{2} & $\otimes$ & $\otimes$ & 8 & 8 & $\theta$ & $\theta$ \\
\hline & 1 & Cytoplasmic & 0 & 0 & OUT: 0 & \\
\hline \multirow[t]{2}{*}{3} & 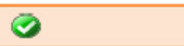 & 6 & 0 & 6 & 6 & ? \\
\hline & 0 & OuterMembrane & 1 & 3 & OUT: 0 & \\
\hline \multirow[t]{2}{*}{4} & 0 & $\theta$ & $\times$ & 8 & 8 & 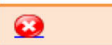 \\
\hline & 0 & OuterMembrane & 0 & 15 & OUT: 0 & \\
\hline \multirow[t]{2}{*}{5} & $\varnothing$ & 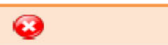 & $\varnothing$ & 8 & $\Leftrightarrow$ & $Q$ \\
\hline & 0 & $\begin{array}{l}\text { CytoplasmicMem } \\
\text { brane }\end{array}$ & 1 & 0 & IN: 8 & \\
\hline \multirow[t]{2}{*}{6} & 8 & 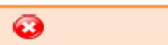 & 8 & 8 & 8 & $\underline{x}$ \\
\hline & 0 & $\begin{array}{l}\text { CytoplasmicMem } \\
\text { brane }\end{array}$ & 0 & 0 & $\mathbb{I N}: 2$ & \\
\hline \multirow[t]{2}{*}{7} & 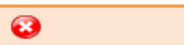 & 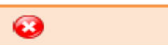 & $\varnothing$ & $\varnothing$ & 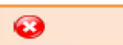 & ? \\
\hline & 2 & $\begin{array}{l}\text { CytoplasmicMem } \\
\text { brane }\end{array}$ & 3 & 1 & $\mathbb{I N}: 6$ & \\
\hline \multicolumn{7}{|c|}{ Meets criteria } \\
\hline \multicolumn{7}{|c|}{ Do not meet criteria } \\
\hline \multicolumn{7}{|c|}{2 Partially annotated proteins } \\
\hline
\end{tabular}

Fig. 3 VacSol-generated results. VacSol generated a summary report for the complete H. pylori proteome with prioritized proteins. Each protein is assigned a unique VacSol ID

\section{Conclusion}

VacSol is a new, highly efficient, and user-friendly pipeline established for biological scientists, including those with limited expertise in computational analyses. VacSol has restricted the pool of promising PVCs from the whole bacterial pathogen proteome by automatizing the in silico reverse vaccinology approach for the prediction of highly antigenic targeted proteins, via a user controlled step-wise process. This new pipeline is an efficient tool in the contexts of time and computational/experimental costs by eliminating false positive candidates from laboratory evaluation. The modular structure of VacSol improves the prediction process of vaccine candidates with additional potential for future development in this field.

\section{Availability and requirements}

Project name: VacSol: An in silico pipeline to predict potential therapeutic targets

Project home page: https://sourceforge.net/projects/ vacsol/files/

Archived version: Not available

Operating system(s): Linux

Programming language: Java

Other requirements (Pre Requisite Tools/Languages):

- PSORTb [17]

- NCBI BLAST+ [38]
- Pftools [39]

- Hmmtop [32]

- ABCPred [40]

- ProPred-I [41]

- ProPred [42]

- Java

- Perl

- Bioperl

\section{Additional files}

Additional file 1: Installation Guide. Description: Detailed user guide for installation and usage of VacSol. (PDF $1178 \mathrm{~kb}$ )

Additional file 2: Test data results. Description: Detailed results of test data (H. pylori) generated by VacSol. (PDF 41330 kb)

\section{Abbreviations}

DEG: Database of Essential Genes; FecA: Iron (III) dicitrate transport protein A; FlaA: Flagelline protein A; FlaB: Flagelline protein B; H. pylori: Helicobacter pylori; HcpA: Helicobacter cysteine-rich protein A; HcpC: Helicobacter cysteine-rich protein C; PVCs: Potential vaccine candidates; VFDB: Virulence factor database

\section{Acknowledgments}

We acknowledge Andreana N. Holowatyj (Ph.D) from Department of Biological Sciences, Wayne State University School of Medicine, USA for proofreading the manuscript.

- Any restrictions to use by non-academics: No

Funding

No funding was provided for this project. 


\section{Availability of data and materials}

VacSol is tested on Ubuntu and can be freely downloaded from: https://sourceforge.net/projects/vacsol/.

The VacSol Installation and User Guide can be obtained from:

https://sourceforge.net/projects/vacsol/files/Installation and User Guide.docx/ download/

H. pylori 26695 dataset used for analysis:

Protein sequences and their locations:

https://drive.google.com/open?id=0B4QOadCkpLvxYXNRbUd3MDF3RHM

H. pylori 26695 full genome:

Full genome was retrieved from NCBI RefSeq with reference number

NC_000915.1, available at following link.

https://www.ncbi.nlm.nih.gov/nuccore/NC_000915.1

\section{Authors' contributions}

AA conceived the idea. MR, JA, AN and AA designed the pipeline. MR implemented the software. AN and MR contributed to software validation. AN and MR composed the manuscript. JA, KN, AO, TP, and MA contributed to analyses and results, as well as in the drafting of the manuscript. All authors read and approved the final manuscript.

\section{Competing interests}

The authors declare that they have no competing interests.

\section{Consent for publication}

Not applicable.

\section{Ethics approval and consent to participate}

Not applicable.

\section{Author details}

${ }^{1}$ Research Center for Modelling and Simulation (RCMS), National University of Sciences and Technology (NUST), H-12, Islamabad, Pakistan. ${ }^{2}$ Atta-ur-Rahman School of Applied Biosciences (ASAB), National University of Sciences and Technology (NUST), H-12, Islamabad, Pakistan. ${ }^{3}$ Biosciences Department, COMSATS Institute of Information Technology, Islamabad, Pakistan.

\section{Received: 27 September 2016 Accepted: 8 February 2017}

\section{Published online: 13 February 2017}

\section{References}

1. Tarca AL, et al. Machine learning and its applications to biology. PLoS Comput Biol. 2007;3(6):e116.

2. Capecchi B, et al. The genome revolution in vaccine research. Curr Issues Mol Biol. 2004;6:17-28.

3. Kimman T. Risks connected with the use of conventional and genetically engineerd vaccines. Vet Q. 1992;14(3):110-8.

4. Rinaudo CD, et al. Vaccinology in the genome era. J Clin Invest. 2009:119(9): 2515-25.

5. Pizza $M$, et al. Identification of vaccine candidates against serogroup $B$ meningococcus by whole-genome sequencing. Science. 2000;287(5459): 1816-20.

6. Rappuoli R. Reverse vaccinology, a genome-based approach to vaccine development. Vaccine. 2001;19(17):2688-91.

7. Ariel $\mathrm{N}$, et al. Search for potential vaccine candidate open reading frames in the Bacillus anthracis virulence plasmid pXO1: in silico and in vitro screening. Infect Immun. 2002;70(12):6817-27.

8. Montigiani $\mathrm{S}$, et al. Genomic approach for analysis of surface proteins in Chlamydia pneumoniae. Infect Immun. 2002;70(1):368-79.

9. Ross BC, et al. Identification of vaccine candidate antigens from a genomic analysis of Porphyromonas gingivalis. Vaccine. 2001;19(30):4135-42.

10. Rappuoli R. Reverse vaccinology. Curr Opin Microbiol. 2000;3(5):445-50.

11. Hassan A, et al. Pangenome and immuno-proteomics analysis of Acinetobacter baumannii strains revealed the core peptide vaccine targets. BMC Genomics. 2016;17(1):732.

12. He $Y$, Xiang Z, Mobley HL. Vaxign: the first web-based vaccine design program for reverse vaccinology and applications for vaccine development. Biomed Res Int. 2010;2010:29750.

13. Doytchinova IA, Flower DR. VaxiJen: a server for prediction of protective antigens, tumour antigens and subunit vaccines. BMC Bioinformatics. 2007; 8(1):4.
14. Jaiswal $V$, et al. Jenner-predict server: prediction of protein vaccine candidates (PVCs) in bacteria based on host-pathogen interactions. BMC Bioinformatics. 2013;14(1):211.

15. Vivona S, Bernante F, Filippini F. NERVE: new enhanced reverse vaccinology environment. BMC Biotechnol. 2006;6(1):35.

16. Goodswen SJ, Kennedy PJ, Ellis JT. Vacceed: a high-throughput in silico vaccine candidate discovery pipeline for eukaryotic pathogens based on reverse vaccinology. Bioinformatics. 2014;30:2381-3.

17. Gardy JL, et al. PSORTb v. 2.0: expanded prediction of bacterial protein subcellular localization and insights gained from comparative proteome analysis. Bioinformatics. 2005;21(5):617-23.

18. Petty DR, Sylvester SA. Specification language for defining user interface panels that are platform-independent. 2002. Google Patents.

19. Baltrus DA, et al. The complete genome sequence of Helicobacter pylori strain G27. J Bacteriol. 2009:191(1):447-8.

20. Kotb M. Infection and autoimmunity: a story of the host, the pathogen, and the copathogen. Clin Immunol Immunopathol. 1995;74(1):10-22.

21. Naz A, et al. Identification of putative vaccine candidates against Helicobacter pylori exploiting exoproteome and secretome: a reverse vaccinology based approach. Infect Genet Evol. 2015;32:280-91.

22. Pruitt KD, Tatusova T, Maglott DR. NCBI reference sequences (RefSeq): a curated non-redundant sequence database of genomes, transcripts and proteins. Nucleic Acids Res. 2007;35 suppl 1:D61-5.

23. Boeckmann B, et al. The SWISS-PROT protein knowledgebase and its supplement TrEMBL in 2003. Nucleic Acids Res. 2003;31(1):365-70.

24. Pertsemlidis A, Fondon JW, John W. Having a BLAST with bioinformatics (and avoiding BLASTphemy). Genome Biol. 2001;2(10):1-10.

25. Kaufmann SH, Lambert PH. The grand challenge for the future: vaccines for poverty-related diseases from bench to field. Springer Science \& Business Media; 2005. http://www.springer.com/br/book/9783764371753.

26. Grandi G. Bacterial surface proteins and vaccines. Ital J Biochem. 2007;56(3):R.

27. Yu C-S, et al. CELLO2GO: a Web server for protein subCELlular LOcalization prediction with functional gene ontology annotation. PLoS One. 2014;9(6): e99368.

28. Luo $\mathrm{H}$, et al. DEG 10, an update of the database of essential genes that includes both protein-coding genes and noncoding genomic elements. Nucleic Acids Res. 2013:42(D1):D574-D80.

29. Handman E. Leishmaniasis: current status of vaccine development. Clin Microbiol Rev. 2001;14(2):229-43.

30. Muhammad SA, et al. Prioritizing drug targets in Clostridium botulinum with a computational systems biology approach. Genomics. 2014;104(1):24-35.

31. Chen $L$, et al. VFDB: a reference database for bacterial virulence factors. Nucleic Acids Res. 2005;33 suppl 1:D325-8.

32. Tusnady GE, Simon I. The HMMTOP transmembrane topology prediction server. Bioinformatics. 2001;17(9):849-50.

33. Consortium U. The universal protein resource (UniProt). Nucleic Acids Res. 2008;36 suppl 1:D190-5.

34. Hsieh P-F, et al. Serum-induced iron-acquisition systems and TonB contribute to virulence in Klebsiella pneumoniae causing primary pyogenic liver abscess. J Infect Dis. 2008;197(12):1717-27.

35. Constantinescu C, Constantinescu E. Could knowledge of H. pylori pathogenicity factors lead to the emergence of new methods for identifying bacteria? Bull Transilvania University of Brasov, Seriels Vl: Medical Sciences. 2014;7:1

36. Mittl PR, et al. Detection of high titers of antibody against Helicobacter cysteine-rich proteins A, B, C, and E in Helicobacter pylori-infected individuals. Clin Diagn Lab Immunol. 2003:10(4):542-5.

37. Mittl PR, Schneider-Brachert W. Sel1-like repeat proteins in signal transduction. Cell Signal. 2007:19(1):20-31.

38. Camacho C, et al. BLAST+: architecture and applications. BMC Bioinformatics. 2009:10(1):421.

39. Sigrist CJ, et al. PROSITE: a documented database using patterns and profiles as motif descriptors. Brief Bioinform. 2002;3(3):265-74.

40. Saha S, Raghava G. Prediction of continuous B-cell epitopes in an antigen using recurrent neural network. Proteins. 2006;65(1):40-8.

41. Singh H, Raghava G. ProPred1: prediction of promiscuous MHC Class-I binding sites. Bioinformatics. 2003;19(8):1009-14.

42. Singh H, Raghava G. ProPred: prediction of HLA-DR binding sites. Bioinformatics. 2001;17(12):1236-7. 